\title{
Dehydration affects the quality of perilla
}

\section{Chao Zhang ${ }^{\mathrm{a}}$, Jiangchangmei $\mathrm{Lu}^{\mathrm{b}}$, Yubin $\mathrm{Wang}^{\mathrm{c}}$, Yue $\mathrm{Ma}^{\mathrm{d}}$, Xiaoyan Zhao ${ }^{\mathrm{e}}$}

Beijing Vegetable Research Center, Beijing Academy of Agriculture and Forestry Sciences; Beijing Key Laboratory of Fruits and Vegetable Storage and Processing; Key Laboratory of Biology and Genetic Improvement of Horticultural Crops (North China), Ministry of Agriculture; Key Laboratory of Urban Agriculture (North), Ministry of Agriculture, 9 Shuguanghuayuan Road, Haidian District, Beijing, China

azhangchao@nercv.org, ${ }^{b}$ lujiangchangmei@nercv.org, 'wangyubin@nercv.org, ${ }^{\mathrm{d}}$ mayue@nercv.org , 'zhaoxiaoyan@nercv.org

Keywords: perilla, dehydration, drying temperature, water activity

Abstract. The effect of dehydration on the quality of perilla was evaluated. The moisture content and water activity of the perilla decreased with the drying time and temperature. The chlorophyll content was enhanced when the drying temperature was decreased. The microstructure and cell wall were destroyed when the drying temperature was enhanced. Hence, a lower drying temperature was better to hold the quality of perilla.

\section{Introduction}

Perilla (Perilla frutescens L.) is rich in protein, vitamins and minerals, and rosmarinic acid, polyphenols, flavonoids, perilla aldehyde, $\alpha$-linolenic acid and other biologically active substances [1]. Recently, considerable attentions have been given to the health benefits of perilla, such as anti-allergic [2], anti-oxidation, anti-cancer [3], anti-tumor [4], antibacterial [5], and anti-HIV [6]. Consequently, perilla is widely welcomed due to its attractive color and flavor, high in medicinal value. Dehydrated perilla are consumed due to its convenience and specific flavor. However, researches about perilla were mainly focused on the essential oil, polyphenol, and functionality of perilla [7-9]. The dehydration processing of the perilla was neglected. Hence, the effect of dehydration on the quality of perilla was evaluated.

\section{Material and Methods}

Dehydration of perilla. Perilla was picked from our Tongzhou farm (Tongzhou District Beijing, 2014). The fresh leaf of the perilla was stored at $4{ }^{\circ} \mathrm{C}$ before use. The perilla was washed by the tap water at $4{ }^{\circ} \mathrm{C}$ to remove the soil and some inclusion. The washed perilla was heated at $55,65,75,85$ and $95{ }^{\circ} \mathrm{C}$ in a air-circulated oven respectively. The moisture content of $7 \%$ was the termination for each treatment. During each treatment, about 7 samples were picked up for the quality determination. The fresh perilla was designated as the control.

Determination of chlorophyll by spectrophotometry. To measure the chlorophyll content, 20 $\mathrm{ml}$ of $80 \%$ acetone was added to $5 \mathrm{~g}$ of each sample and mixed with a homogenizer (IKA T10 Basic, Werke GmbH \& Co. KG, Staufen, Germany) for $1 \mathrm{~min}$ at a high speed, then centrifuged at $8,000 \mathrm{rpm}$ for $5 \mathrm{~min}$ at $4{ }^{\circ} \mathrm{C}$, and then filtered through Whatman No. 1 filter papers. The volume was adjusted to $25 \mathrm{~mL}$ and the absorbance values were measured at 663 and $645 \mathrm{~nm}$ by a spectrophotometer (UV-1800, Shimadzu Corporation, Kyoto, Japan). The chlorophyll content of the sample was calculated followed by Equation 1 .

$$
\text { Chlorophyll }=\frac{\left(20.2 \times A_{645}+8.02 \times A_{663}\right) \times D}{1000}
$$

where chlorophyll content is $\mathrm{mg} / \mathrm{g}$ fresh weight; $A_{645}$ and $A_{663}$ are the absorbance at $645 \mathrm{~nm}$ and $663 \mathrm{~nm}$, respectively; $D$ is the dilution factor of the sample.

Moisture content determination. The moisture content of the sample was measured by heating at $105{ }^{\circ} \mathrm{C}$ until a constant weight was reached. The moisture content was the percentage of the 
weight loss in the total weight.

Water activity determination. The water activity of the sample was measured by a Aqua LAB 4TE (Decagon Devices, USA). The Aqua LAB 4TE was calibrated by the water activity standard solution of 1.0, 0.75 and 0.5 respectively before measurement. The sample was powdered and was covered on the sample plate. The data was recorded when the result was constant.

Microstructure. Microstructure of the samples was imaged by a scanning electron microscopy (S-4800, Hitachi Co., Japan). The specimens were attached with a double side tape and sputtered with gold. The cross-section and surface of the specimens were captured at an acceleration voltage of $5 \mathrm{kV}$. The perilla that was frozen dried was named as the control.

Statistical Analysis. Analysis of variance (ANOVA) was used to compare mean differences of the results. If the differences in mean existed, multiple comparisons were performed using Duncan’s Multiple Range Test. All analysis was conducted using SPSS for Window Version 19. All experiments were done in triplicates or more.

\section{Results and Discussion}

Effect of dehydration on moisture content of perilla. The effect of dehydration on the moisture content of perilla is shown in Figure 1.The moisture content decreased with the drying time. The decrease was able to be divided into 3 stages. Firstly, the moisture content decreased slowly, and then decreased quickly. Finally, the moisture content reached to a constant level of $2 \% \sim 7 \%$. The drying temperature affected the drying duration. A higher temperature reduced the drying time. For example, the drying at $55{ }^{\circ} \mathrm{C}$ cost 270 min to reach the moisture content of $7 \%$, while drying at $95{ }^{\circ} \mathrm{C}$ only cost $70 \mathrm{~min}$. Similar results also presented in the dehydration of cabbage [10], carrot [11], and mint [12].

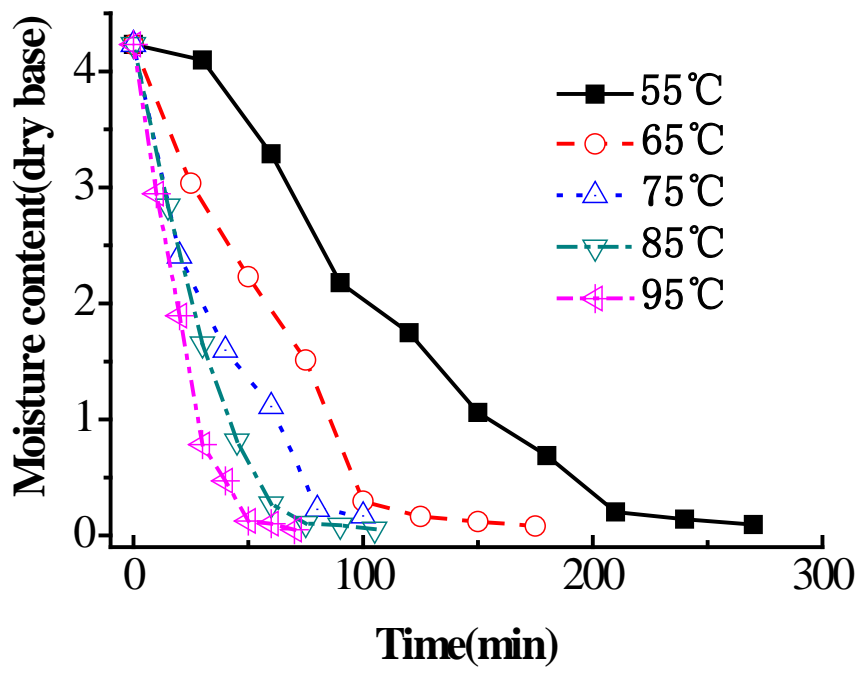

Figure 1 Effect of dehydration on moisture content of perilla

Effect of dehydration on water activity of perilla. Water activity is an important consideration for the sensory properties and safety of foods, which is defined as the vapor pressure of a liquid divided by that of pure water at the same temperature [13]. Nearly no microorganisms survive when the water activity is below 0.60 , and the growth of the most bacteria, yeast, and fungi is inhibited when the water activity is below 0.85 [14]. The effect of dehydration on the water activity of perilla is shown in Figure 2. The water activity decreased with the drying time. Meanwhile, a higher temperature accelerated the decrease of the water activity. The water activity of 0.60 has been regard as a better criterion of the dehydrated cabbage [10]. Based on the Figure 1 and Figure 2, the water activity of the dehydrated perilla was lower than 0.6 when the moisture content of $7 \%$ was reached. Consequently, the moisture content of $7 \%$ leaded to an over-drying of the perilla. 


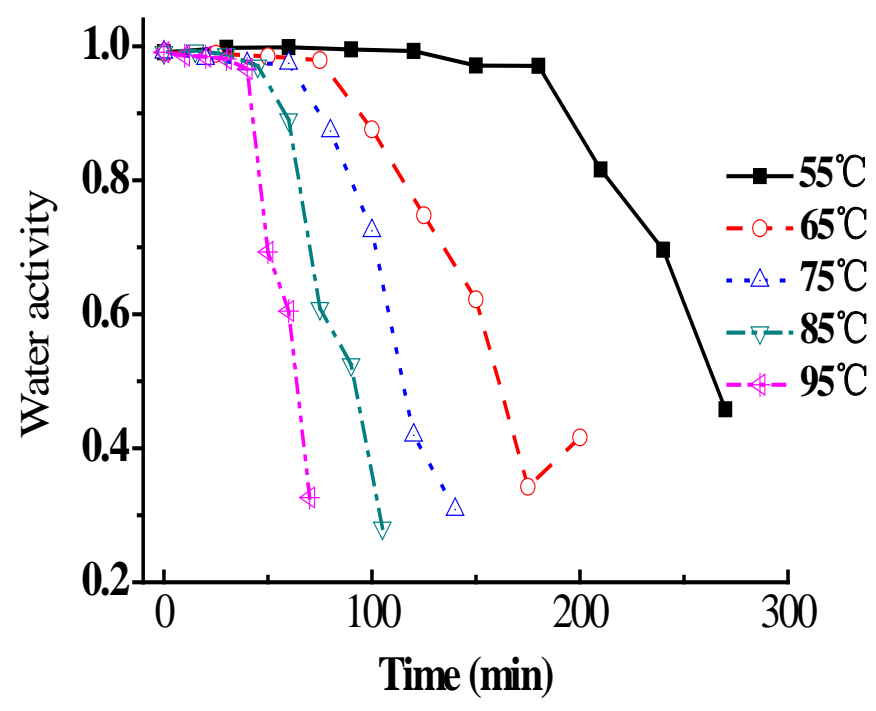

Figure 2 Effect of dehydration on water activity of perilla

Effect of dehydration on chlorophyll content of perilla. Chlorophylls are susceptible to physical, chemical or enzymatic degradation reactions [15]. The simultaneous actions of enzymes, oxygen, light and heat lead to the conversation from chlorophyll $\rightarrow$ pheophytin $\rightarrow$ pheophorbide. The rapid conversion of chlorophyll to pheophytin suggests rapid acidic removal of $\mathrm{Mg}^{2+}$ upon addition of dressing, whereas the rather slow conversion of pheophytin to pheophorbide suggests an enzymatic reaction that is mediated chlorophyllase [16]. The effect of drying temperature on the chlorophyll content of perilla is shown in Figure 3. The chlorophyll content decreased with the drying time. Meanwhile, a higher temperature accelerated the degradation of the chlorophyll in the perilla. The chlorophyll degradation of cabbages [17] and spinach [18] has been proved to follow the first-order-kinetics reaction. The degradation rate was highly exponential with temperature, thereby the degradation rate at a low temperature was much lower than that at a high temperature. A lower temperature was more effective to hold the chlorophyll content of the perilla.

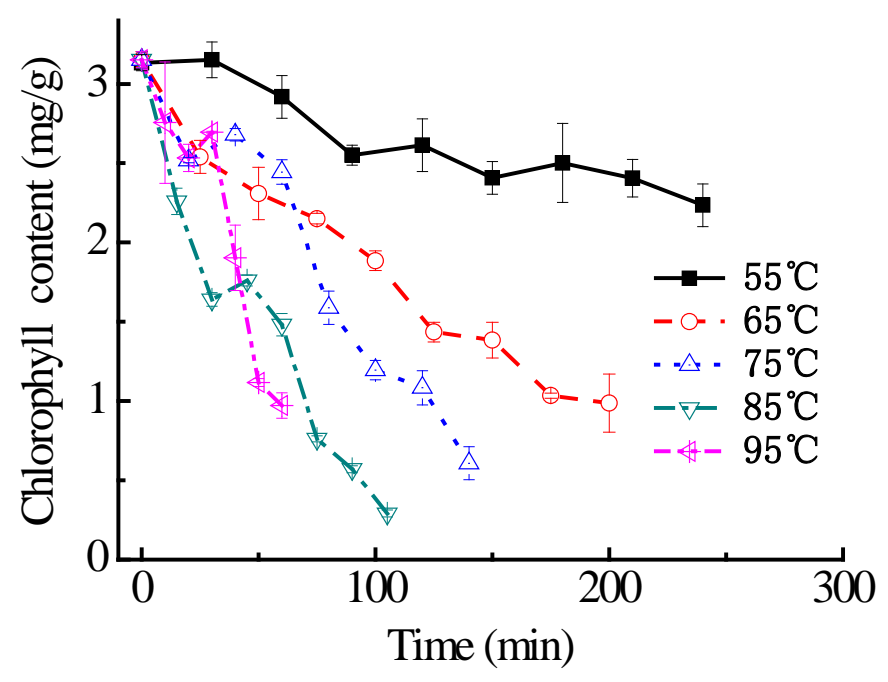

Figure 3 Effect of dehydration on chlorophyll content of perilla

Effect of dehydration on microstructure of perilla. The effect of drying temperature on cross-section of perilla is shown in Figure 4. The cross section of the control was tousy with a smooth cell wall, while that of the dehydrated perilla was compressed with a shrink cell wall. Moreover, the cell wall of the perilla was clear when being heated at $55{ }^{\circ} \mathrm{C}$, while that was blurry when being heated at 65, 75, 85 and $95{ }^{\circ} \mathrm{C}$. Hence, a lower temperature was more effective to hold 
the microstructure of the dehydrated perilla.
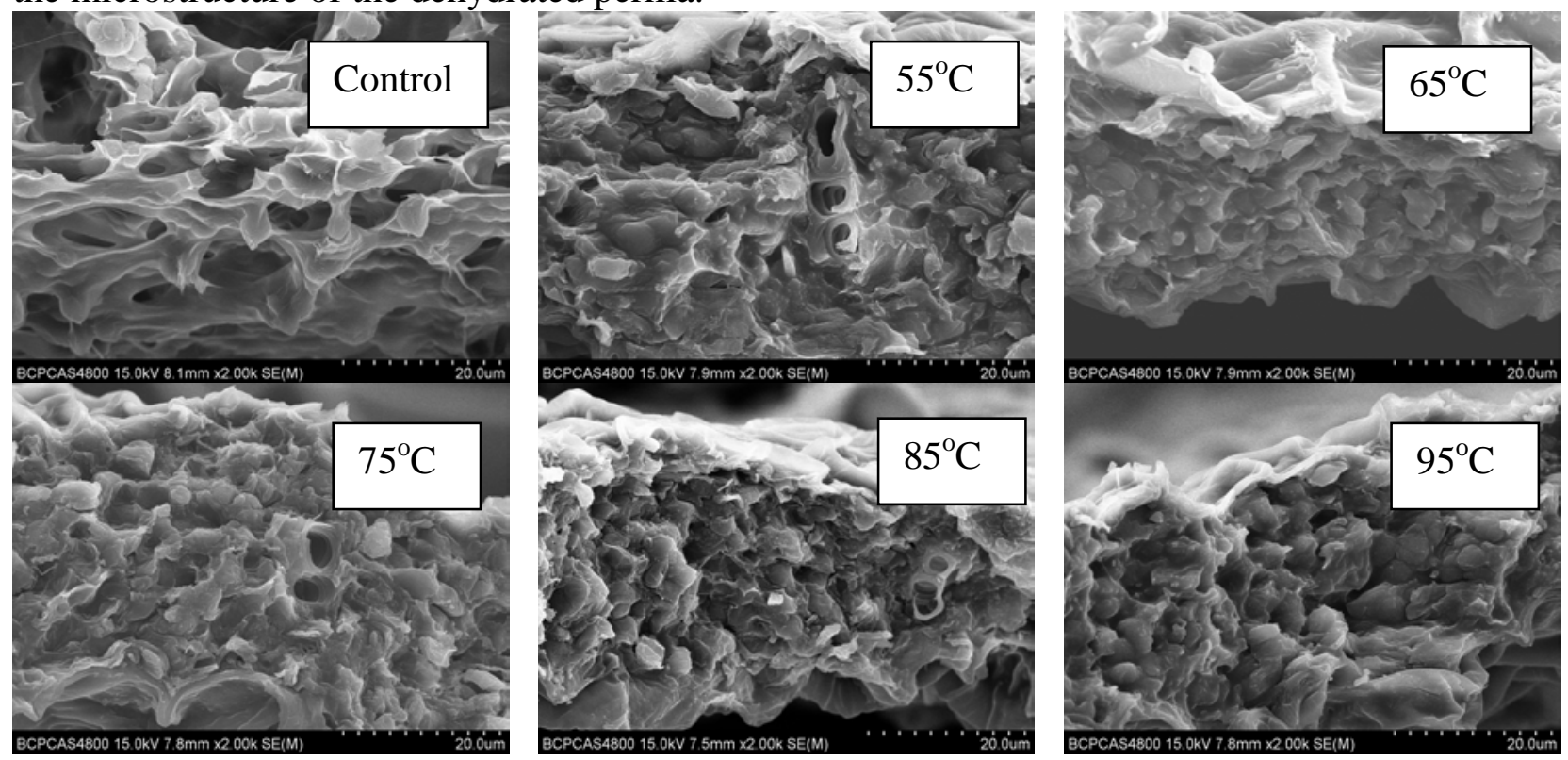

Figure 4 Effect of dehydration on cross-section of perilla

\section{Conclusions}

The moisture content and water activity of the perilla decreased with the drying time and drying temperature. The chlorophyll content was enhanced when the drying temperature was decreased. The microstructure and cell wall were destroyed when the drying temperature was high. Hence, a lower drying temperature was better to hold the quality of perilla.

\section{Acknowledgements}

The authors are grateful to financial support of the earmarked fund for Modern Agro-industry Technology Research System (CARS-26-22 \& CARS -25) and Beijing Academy of Agricultural and Forestry Sciences, New Discipline Breeding (KJCX20140204).

\section{References}

[1] L. Meng, Y. Lozano, I. Bombarda, E. M. Gaydou, B. Li: Compt. Rendus Chimie Vol 12 (2009), p. 602.

[2] R. Guo, M. H. Pittler, E. Ernst: Ann. Allergy Asthma Immunol. Vol 99 (2007), p. 483.

[3] N. Banno, T. Akihisa, H. Tokuda, K. Yasukawa, H. Higashihara, M. Ukiya: Biosci. Biotechnol. Biochem. Vol 68 (2004), p. 85.

[4] H. Ueda, C. Yamazaki, M. Yamazaki: Biol Pharmaceutical Bull Vol 26 (2003), p. 560.

[5] H. Yamamoto, T. Ogawa: Biosci Biotechnology and Biochemistry Vol 66 (2002), p. 921.

[6] T. Kawahata, T. Otake, H. Mori, Y. Kojima, I. Oishi, S. Oka: Antiviral Chem. Chemothera. Vol 13 (2002), p. 283.

[7] I. Gülçin, D. Berashvili, A. Gepdiremen: J. Ethnopharmacol. Vol 101 (2005), p. 287.

[8] J. W. Wang, Z. H. Xia, J. H. Chu, R. X. Tan: Enzyme Microb. Technol. Vol 34 (2004), p. 651.

[9] C. You, Y. Wang, W. Zhang, K. Yang, Y. Wu, Z. Geng, H. Chen, H. Jiang, S. Du, Z.. Deng, Z. Liu: Industr. Crops Prod. Vol 61 (2014), p. 331.

[10]D. Wang, L. Jiang, X. Zhao, C. Zhang, Y. Ma: Adv Mate Res Vol 396-398 (2012), p. 1297. 
[11] M. A. Goula, G. K. Adamopoulos: Drying Technol Vol 28 (2010), p. 752.

[12]M. C. Díaz-Maroto, M. S. Pérez-Coello, M. A. G. Viñas, M. D. Cabezudo: J. Agricul. Food Chem.. Vol 51 (2003), p. 1265.

[13]S. S. Sablani, S. Kasapis, M. S. Rahman: J. Food Eng. Vol 78 (2007), p. 266.

[14]V. Bohm, N. L. Putpitasarı-Nienaber, M. G. Ferruzzi, S. J. Schwartz: Food Chem. Vol 50 (2002), p. 221.

[15]N. Koca, F. Karadeniz, H. S. Burdurlu: Food Chem. Vol 100 (2006), p. 609.

[16]W. J. Heaton, Y. R. Yada, G. A. Marangoni: J. Agricul. Food Chem. Vol 44 (1996), p. 395.

[17]D. M. Wang, L. Z. Jiang, X. Y. Zhao, C. Zhang, Y. Ma: Adv. Mater. Rese. Vol 396-398 (2011), p. 1297.

[18]F. M. Lajolo, U. M. Lanfer Marquez: J.Food Sci. Vol 47 (1982), p. 1995. 